\title{
Precision Scale Determination from the Upsilon Spectrum
}

\section{lain D. Kendall*, C. T. H. Davies, C. Mcneile}

Department of Physics and Astronomy, University of Glasgow, Glasgow, UK

E-mail: i.kendallephysics.gla.ac.uk

\section{G. P. Lepage}

LEPP, Cornell University, Ithaca, NY, USA

\section{J. Shigemitsu}

Physics Department, The Ohio State University, Columbus, OH, USA

\section{HPQCD Collaboration ${ }^{\dagger}$}

We determine the radial excitation energy (2S-1S splitting)in the Upsilon spectrum using NRQCD b quarks on MILC configurations. We have statistical fitting errors of around $1 \%$ across a range of lattice spacing values using random wall sources, and this allows us to give improved determinations of the physical value of the heavy quark potential parameter $r_{1}$.

We also show improved results for the hyperfine splitting and the ratio of leptonic widths of the $\Upsilon$ and $\Upsilon^{\prime}$.

The XXVI International Symposium on Lattice Field Theory

July 14 - 19, 2008

Williamsburg, Virginia, USA

\footnotetext{
* Speaker.

${ }^{\dagger}$ http://www.physics.gla.ac.uk/HPQCD/
} 


\section{Determining Lattice Scales}

We find the lattice spacing, $a$, on a series of MILC [1] ensembles by calculating the spectrum of the $\Upsilon$ (upsilon) particle and defining the spacing as the ratio of the lattice value over the experimental value of the $2 \mathrm{~S}-1 \mathrm{~S}$ energy splitting. The $\Upsilon$ is chosen because the $\Upsilon 2 \mathrm{~S}-1 \mathrm{~S}$ splitting is known from experiment to be insensitive to the valence heavy quark mass.

This gives us values of $a$ particular to the ensembles we used in our calculation, but we can make this more generally useful by use of the heavy quark potential scale parameter known as $r_{1}$. The $r_{1}$ parameter is defined as the value of $r$ at which $r^{2} F(r)=1$ where $F$ is the gradient of the heavy quark potential. It is not a physically meaningful quantity, and is not something that can be measured by experiment, however it is quantity that lends itself to calculation on the lattice, and indeed $r_{1} / a$ is one of the first things calculated by the MILC collaboration on the ensembles that they generate.

\begin{tabular}{|l|l|l|l|l|l|}
\hline Lattice & \multicolumn{2}{|c|}{ V. Coarse } & \multicolumn{2}{c|}{ Coarse } & Superfine \\
\hline Size & $16^{3} \times 48$ & $16^{3} \times 48$ & $20^{3} \times 64$ & $24^{3} \times 64$ & $48^{3} \times 144$ \\
$n_{f}$ & $2+1$ & $2+1$ & $2+1$ & $2+1$ & $2+1$ \\
$\beta$ & 6.572 & 6.586 & 6.760 & 6.760 & 7.470 \\
$u_{0 P} a m_{u, d}$ & 0.0097 & 0.0194 & 0.010 & 0.005 & 0.0036 \\
$u_{0 P} a m_{s}$ & 0.0484 & 0.0484 & 0.050 & 0.050 & 0.018 \\
$u_{0 L}$ & 0.8218 & 0.8225 & 0.8359 & 0.8362 & 0.8695822 \\
$a M_{b}^{0}$ & 3.40 & 3.40 & 2.80 & 2.80 & 1.34 \\
$n$ & 4 & 4 & 4 & 4 & 4 \\
Configs & 631 & 631 & 595 & 202 & 132 \\
Origins & 24 & 24 & 32 & 32 & 8 \\
\hline
\end{tabular}

Table 1: Summary of MILC ensemble parameters[1] Sea quark masses are given using the MILC convention which includes a factor of $u_{0}$ obtained from the plaquette. We also give values of $u_{0}$ from the Landau gauge link which we use in the NRQCD action. Origins are the number of different starting times used on each lattice. $\mathrm{n}$ is the NRQCD stability parameter, see [2]. The value of 4 is different from our previous work which used 2[3]. The higher value is needed for the superfine ensemble, and it is beneficial to use a consistent value across all ensembles to keep discretisation errors comparable. 'Configs' is the number of configurations used in this work, and may not represent the total number of configurations in existence for that ensemble.

We use our determination of $a$ on particular ensembles along with the MILC determination of the $r_{1} / a$ parameter on these ensembles to give a value of $r_{1}$ in physical units. This value, once extrapolated to the continuum limit (i.e. $a \rightarrow 0$ ), is a 'real world' value which, in principle, is independent of ensemble. The value of $r_{1}$ can be combined with the $r_{1} / a$ values of other ensembles to define their values of $a$. Thus our scale determination is no longer specific to the ensembles we used, and is portable to other lattice ensembles.

The improvement of the error in this quantity is significant because the uncertainty in the lattice spacing contributes to the error of any physical lattice result, in some cases to powers greater than 1 , and can dominate the overall error in such a result. This is the case for several of our high precision calculations, for example our $\pi, K, D, D_{s}$ decay constants paper [4]. 
We use the NRQCD action for our valence b-quarks (see [2]). Our valence quarks are applied to the lattice using a random wall: a random phase associated with the each point of the lattice, which effectively allows us to produce a meson centred on every spatial point of the lattice at the chosen start time. We also utilise various smearing functions to optimise the signal for the states we are interested in, for example to pick out the $2 \mathrm{~S}$ energy level. The $2 \mathrm{~S}$ smearing is chosen to be a function that has a large amplitude for the $2 \mathrm{~S}$ energy, and more importantly has a very small amplitude for the $1 \mathrm{~S}$. The use of these "all-to-all" mesons improves the usage of the available ensembles, while the smearings applied to them allow us to concurrently fit multiple correlators, yielding a better fit.

We have found that the random wall method yields very precise results for the ground state (1S) energy, with errors $\sim 10$ times smaller than those produced without the random wall. In contrast, we have found little improvement for the $2 \mathrm{~S}$ energy when comparing the random wall with smearing function to the smearing function alone.

\section{Results}

\subsection{Lattice Scale Determination}

We calculated a physical $r_{1}$ value on the 5 MILC ensembles mentioned using $r_{1} / a$ values from MILC [5], and the values are plotted below (Figure 1). With the exception of the Superfine ensembles, the errors in these values are all at the $\sim 1 \%$ level. This compares favourably with the existing determination of $0.321(5) \mathrm{fm}$ [3], which is $1.5 \%$.

Within our errors, we see no significant change in $r_{1}$ as a function of either $a$ or $m_{\text {light }}$.

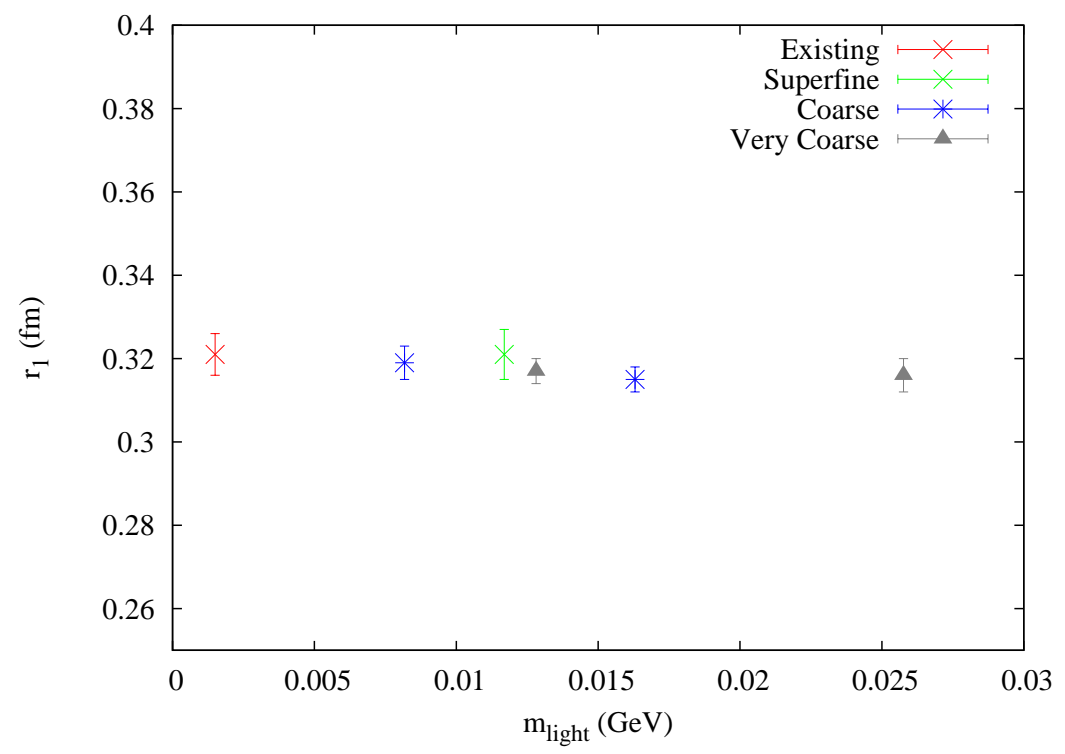

Figure 1: $r_{1}$ plotted against $m_{\text {light }} . m_{\text {light }}$ includes a factor of $u_{0 P}$ from the MILC mass convention as in Table 1 


\subsection{Leptonic Width}

The ratio of leptonic widths of the $\Upsilon^{\prime}$ and $\Upsilon$ can easily be calculated on the lattice. By taking the ratio, factors such as the $Z_{\text {match }}$ renormalisation cancel, making it easier to obtain a precise determination.

To find this ratio, we need matrix elements of the vector current between the appropriate $\Upsilon$ states and the vacuum. This is, at leading order in the relativistic expansion of the currents, simply the amplitudes of the $\delta$-smearing in these $\Upsilon$ states, which is equivalent to the wavefunction at the origin in a potential model $-\Psi_{n}(0)$. For the $2 \mathrm{~S}: 1 \mathrm{~S}$ ratio, we then have:

$$
\frac{\Gamma_{e e}(2 S) M_{\Upsilon(2 S)}^{2}}{\Gamma_{e e}(1 S) M_{\Upsilon(1 S)}^{2}}=\frac{\left|\Psi_{2}(0)\right|^{2}}{\left|\Psi_{1}(0)\right|^{2}}
$$

The results from this can be seen in Figure 2. We see some dependence on $a^{2}$ in Figure 2, however the results at the same lattice spacing are consistent with each other despite the differences in the light quark mass. These results appear to be heading towards consistency with the experimental result as $a \rightarrow 0$.

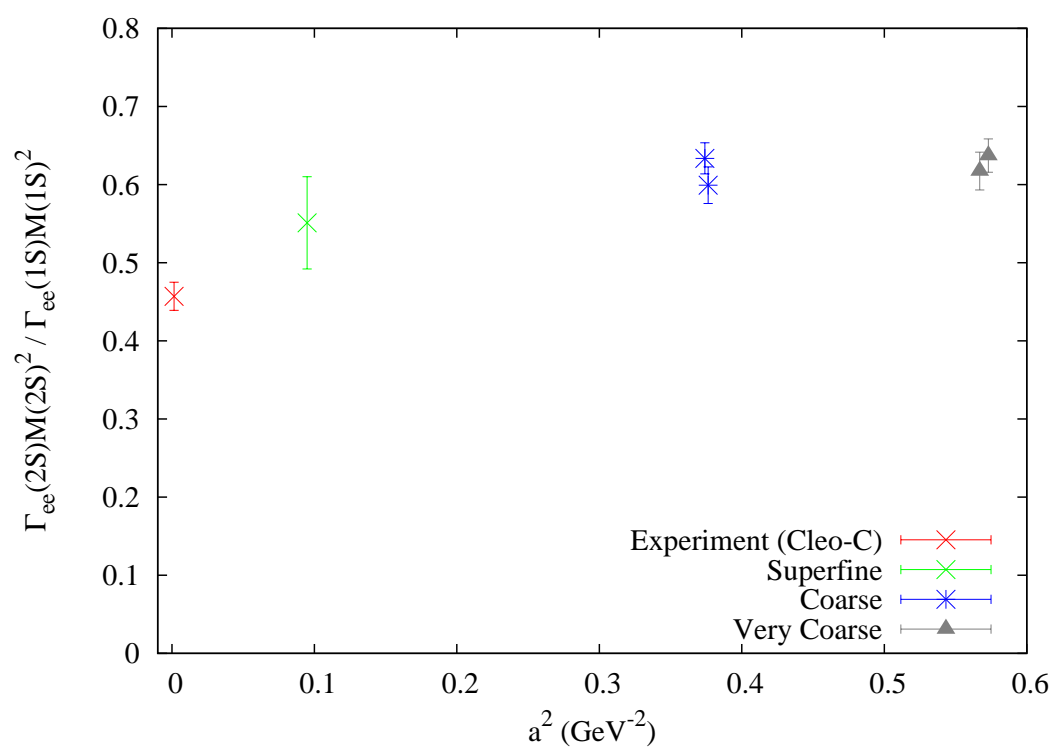

Figure 2: Leptonic width vs. the square of the lattice spacing. Experiment is from [6, 7]

\subsection{Kinetic Masses}

The mass of the $\Upsilon$ cannot be extracted directly from the calculation because the zero of energy in NRQCD is offset. We must instead use $\Upsilon$ particles with momentum to extract the mass using the following relation based on the standard $E^{2}=M^{2}+p^{2}$ equation, where $d E$ is an arbitrary energy offset:

Using the kinetic mass, we can tune the b-quark masses that we input at the start of a calculation until we get a consistent result. For the coarse ensembles, we obtained $M_{\Upsilon}^{\text {kin }}=9.48(6) \mathrm{GeV}$, 


$$
\begin{aligned}
& E_{0}+d E=M \\
& E_{p}+d E=\sqrt{M^{2}+p^{2}} \\
& \text { so }\left(E_{p}-E_{0}+M\right)^{2}=(\Delta E+M)^{2}=M^{2}+p^{2}, \text { giving: } \\
& M=\left(p^{2}-\Delta E^{2}\right) / 2 \Delta E
\end{aligned}
$$

and for the very coarse we obtained $9.45(7) \mathrm{GeV}$, the dominant error is from the uncertainty in $r_{1}$. The experimental result is $9.46030(26) \mathrm{GeV}$ [7].

\subsection{Hyperfine Splitting}

The hyperfine splitting in the $\Upsilon$ system (i.e. the $\Upsilon(1 S)$ and the $\eta_{b}(1 S)$ mass difference) has been notoriously difficult to measure experimentally. Its value was predicted from full lattice QCD, albeit with a 25\% error, by the HPQCD collaboration in 2005 [3]. The new experimental result from BaBar agrees with this prediction [8]. Here, we endeavour to improve on this result, and compare to the new experimental result from the BABAR collaboration [8].

We see little dependence on the light quark mass. This is expected as long as the quark masses are not excessive (i.e they need not be correct, they must simply be 'light'). There is, however, strong $a$ dependence (Figure 3), and this limits how well one can make a determination of a result in the continuum. The error in the previous theoretical result is largely caused by this, and we expect a better result from this work, especially since we have results from ensembles closer to the continuum than before.

The error in the lattice data points includes the error from our $a^{-1}$ determination and statisti$\mathrm{cal} /$ fitting errors in the lattice-unit energy splitting. It does not, however, include systematic errors such as radiative and discretisation errors in the NRQCD action. This requires further work.

\section{The Foo Particle: Checking Systematics}

The 'Foo' particle is an unphysical particle that was contrived as a check of the discretisation errors in the NRQCD action.

The Foo has been designed to be larger than the $\Upsilon$ particle, and as such it is less sensitive to the lattice spacing. Any discrepancy in a comparison between the Foo and the $\Upsilon$ can then be attributed to discretisation errors in the $\Upsilon$. To achieve this, the artificial valence 'quarks' that we use to construct a Foo are given a lower mass than the valence b-quarks used in the $\Upsilon$.

The Foo has also been designed to have no spin structure, so all spin-dependent terms are removed from the action. We keep only a simple $P^{2} /(2 M)$ Hamiltonian with corrections for discretisation errors. The spin terms are unimportant in an already unrealistic particle, and their removal improves the speed at which the particle can be computed. Furthermore, this aspect of the particle, along with the lower valence 'quark' mass, allows for a relatively straightforward perturbation theory calculation along the lines of [9] which can be used to determine the radiative corrections for the remaining coefficients (currently both set to 1) in the modified NRQCD action applied to the Foo. Work is in progress on this, and on the associated numerical calculations of the Foo spectrum. 


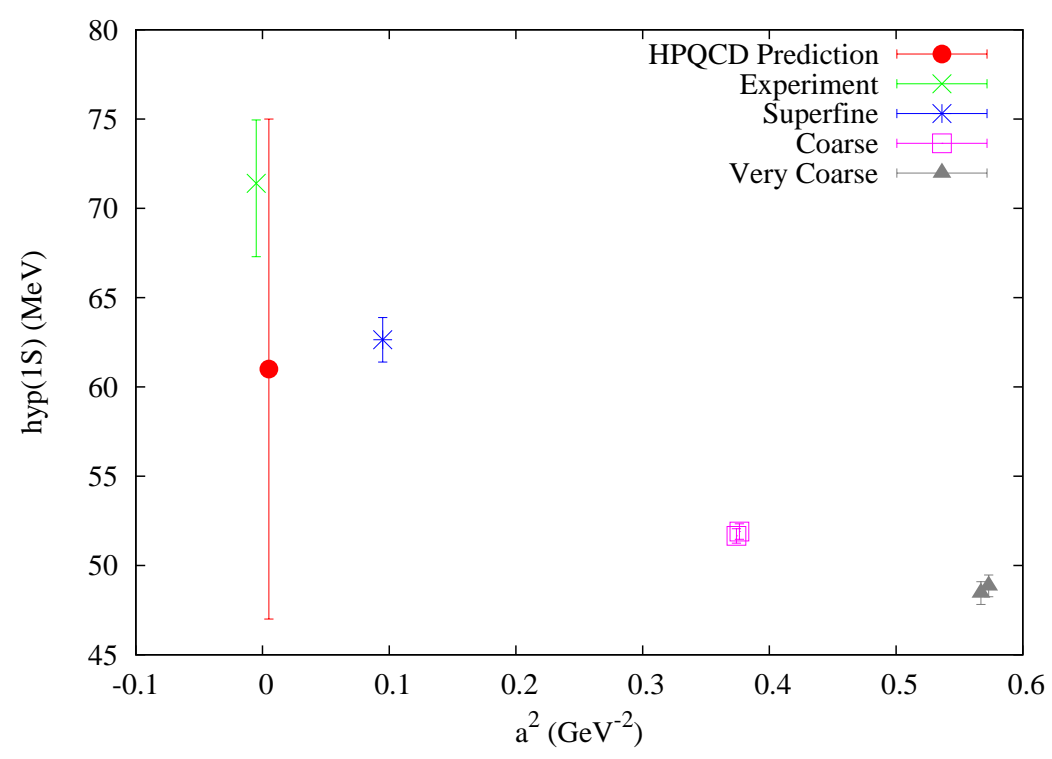

Figure 3: Hyperfine splitting against lattice spacing squared. Experiment is from the BABAR collaboration[8]. (Continuum theory and experimental result offset on the x-axis for clarity.) Results at different $m_{\text {light }}$ are included for the coarse and very coarse ensembles.

\section{Conclusions}

Through this work, it is likely that we will be able to update our physical value for $r_{1}$ with increased precision. Our preliminary results have approximately $1 \%$ errors and cover a larger range of lattice spacing values than our previous calculation. Our preliminary results for the hyperfine splitting and $\Upsilon^{\prime} / \Upsilon$ leptonic widths also look promising.

\section{Acknowledgements}

I would like to thank MILC for the use of their configurations and unpublished $r_{1} / a$ values.

\section{References}

[1] C. W. Bernard et al., "The QCD spectrum with three quark flavors," Phys. Rev. D64 (2001) 054506, arXiv:hep-lat/0104002.

[2] G. P. Lepage, L. Magnea, C. Nakhleh, U. Magnea, and K. Hornbostel, "Improved nonrelativistic QCD for heavy quark physics," Phys. Rev. D46 (1992) 4052-4067, arXiv: hep-lat/ 9205007.

[3] A. Gray et al., "The Upsilon spectrum and m(b) from full lattice QCD," Phys. Rev. D72 (2005) 094507, arXiv:hep-lat/0507013.

[4] HPQCD Collaboration, E. Follana, C. T. H. Davies, G. P. Lepage, and J. Shigemitsu, "High Precision determination of the pi, K, D and $D_{s}$ decay constants from lattice QCD," Phys. Rev. Lett. 100 (2008) 062002, arXiv:0706.1726 [hep-lat].

[5] C. Aubin et al., "Light hadrons with improved staggered quarks: Approaching the continuum limit," Phys. Rev. D70 (2004) 094505, arXiv: hep-lat/ 0402030. 
[6] CLEO Collaboration, J. L. Rosner et al., "Di-electron widths of the Upsilon(1S,2S,3S) resonances," Phys. Rev. Lett. 96 (2006) 092003, arXiv : hep-ex/ 0512056.

[7] Particle Data Group Collaboration, W. M. Yao et al., "Review of particle physics,” J. Phys. G33 (2006) 1-1232.

[8] BABAR Collaboration, "Observation of the bottomonium ground state in the decay Upsilon(3S) $\longrightarrow>$ gamma eta $b$, , arXiv : 0807.1086[hep - ex].

[9] C. J. Morningstar, "Radiative corrections to the kinetic couplings in nonrelativistic lattice QCD," Phys. Rev. D50 (1994) 5902-5911, arXiv : hep-lat/9406002. 